\title{
PULSOS Y PROBABILIDAD DE OCURRENCIA DE INUNDACIÓN DE LA LAGUNA DE FÚQUENE
}

\author{
Pulses and likelihood of Fúquene Lagoon flood occurrence \\ Jimmy Villamizar Martin ${ }^{1}$, Juan David Jiménez Sánchez ${ }^{1}$, Eduardo Zamudio Huertas ${ }^{2}$ \\ ${ }^{1}$ Tecnólogo en Construcciones Civiles, Universidad Distrital Francisco José de Caldas (Bogotá, \\ Colombia). jvillamizarm@correo.udistrital.edu.co,jdjimenezs@correo.udistrital.edu.co \\ ${ }^{2}$ M.Sc. Universidad Distrital Francisco José de Caldas (Bogotá, Colombia). \\ ezamudioh@udistrital.edu.co
}

(Recibido septiembre 5 de 2017 y aceptado diciembre 25 de 2017)

\begin{abstract}
Resumen
La laguna de Fúquene en los últimos 20 años de manera reiterada ha sido afecta por eventos de inundación. Ante dicha afectación se han visto perjudicadas poblaciones aledañas a la Laguna, adicional causa alteraciones a la biodiversidad y ecosistema de la misma. Con el amino de estudiar los eventos de inundación históricos de la Laguna se evaluaran los registros diarios de los niveles de agua de la Laguna de Fúquene. A partir del análisis de dichos niveles de agua históricos se hayo los pulsos de inundación históricos, frecuencia de inundación, probabilidad de permanecía de la inundación. Se detectó que los niveles más altos de la Laguna coinciden con las épocas de invierno de la región, las cuales son Abril, Mayo, Junio, Julio, Noviembre y Diciembre. Los niveles históricos más altos se presentaron en los años 2006, 2010, 2011 y 2012. Dicha información se provee con el objetivo de orientar a la comunidad aledaña a la laguna o tomador de decisiones (entidades pertinentes) al conocer cuándo y duración de una eventual inundación y a partir de ello, generar los planes de contingencia y ordenamiento territorial.
\end{abstract}

Palabras clave: Amenaza; Laguna; Niveles; probabilidad de ocurrencia; pulsos de inundación; Zonas.

\begin{abstract}
The Lagoon of Fuquene has been repeatedly affected for flood events in the last 20 years. Due to this affectation, populations adjacent to the lagoon have been harmed, causing additional alterations to the biodiversity and ecosystem. With the purpose of studying the historical flood events of the lagoon, the daily records of the water levels of the lagoon of Fuquene will be evaluated. Based on that analysis, the historical flood pulses, flood frequency, flood probability of permanence was found. It was detected that the highest levels in the lagoon coincide with the winter seasons, which are April, May, June, July, November and December. The highest historical levels were presented in 2006, 2010, 2011 y 2012. This information is provided with the objective of guiding the nearby community of the lagoon or decision maker (relevant entities) to know when and duration of a possible flood, and to allow generate contingency plans and land use planning.

Key words: Threat; Lagoon; Levels, probability of occurrence; flood pulses, Zones.
\end{abstract}

\section{INTRODUCCIÓN}

La Laguna de Fúquene es uno de los depósitos de agua más importantes de los Andes del Norte, da origen al río Suarez, principal fuente hídrica del municipio de Chiquinquirá [1-2]. Ante la influencia antrópica (uso del suelo para la ganadería, agricultura y minería), la laguna ha perdido aproximadamente 15.000 hectáreas de su espejo de agua, adicional las aguas que alimentan la laguna cuenta con alta carga de materias en suspensión, lo que ocasiona una pérdida del $50 \%$ de su capacidad de retener agua [3-4]. Dicha perdida se traduce en mayor área inundable cercana a la laguna, afectando las actividades humanas, regulación hídrica, depuración de las 
aguas, la biodiversidad y ecosistema. De forma reiterada en los últimos 20 años las zonas aledañas a la Laguna han sido afectadas por inundaciones, principalmente en los meses más lluviosos del año (los cuales se describen más adelante).

Se realizó un estudio elemental de los pulsos de inundación que ha tenido la laguna desde el año de 1996, evaluando los registros diarios de los niveles de agua. Entiéndase como pulso de inundación la fase entre la inundación y sequia [5] o entre la superación de un nivel de referencia determinado y el termino dicho nivel, ósea cuando la lámina de agua es inferior a ese nivel de referencia. Por lo tanto, el pulso de inundación depende del nivel de referencia que se estipule como amenaza o área inundable adyacente a la Laguna [6-7]. Por ello, el número de pulsos de inundación corresponde al número de veces que se ha inundado el área o zona de amenaza. Como referencia se tomaron las cotas que delimitan cada zona de amenaza clasificada por la CAR (Corporación Autónoma Regional de Cundinamarca) [8]. Se procedió a analizar los niveles diarios históricos, utilizando métodos probabilísticos dando como resultado curvas de permanencia que indican cuantos días continuos puede persistir una alerta por inundación para los diferentes niveles de agua en la Laguna, igualmente se determinaron los meses que tienen mayor probabilidad de inundación en el año.

El estudio se realizó con el objetivo de revelar información ante la inundación los niveles de amenaza, permitiendo identificar; número de pulsos de inundación, frecuencia de inundación, probabilidad de permanecía de la inundación, identificación de los meses que tienen mayor probabilidad de inundación en el año. Información que busca orientar a la comunidad o tomador de decisiones (entidades pertinentes) al conocer cuándo, donde (zona de amenaza) y duración de una eventual inundación y a partir de ello, generar los planes de contingencia y ordenamiento territorial.

En el presente artículo se aportan datos estadísticos e información relevante que pueden ser guía para futuros estudios referentes a impacto ambiental, o variaciones de población, entre otros, quedando al criterio del lector.

\section{2. ÁREA DE ESTUDIO}

La laguna de Fúquene se sitúa en Colombia en el Departamento de Cundinamarca, ubicada en la zona central del valle Ubaté - Chiquinquirá, con una altitud de $2537.72 \mathrm{msnm}$. En la figura 1 se georeferencia la laguna de Fúquene, Actualmente tiene una superficie de 3205.3 Ha, cuenta con una desembocadura: El Río Suárez. El nivel de agua es regulado por la compuerta de Tolón, que se encuentra a 18 kilómetros, aguas abajo del nacimiento [8].

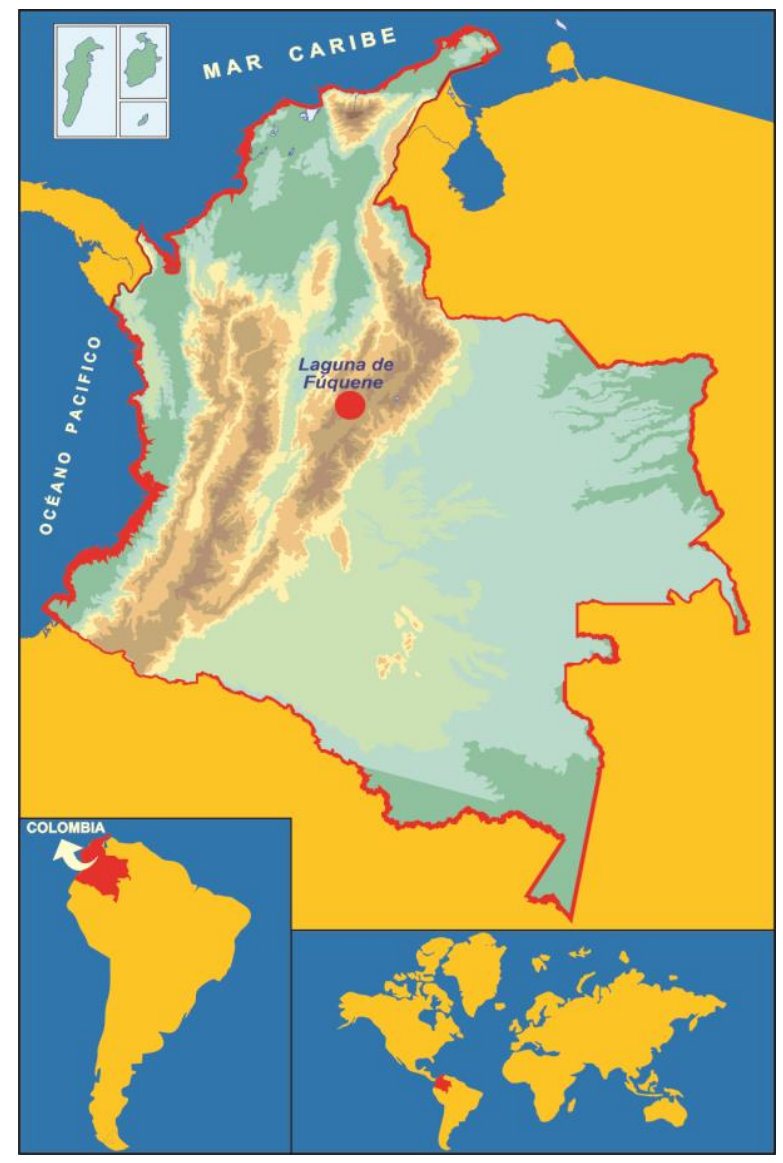

Figura 1. Localización Laguna de Fúquene [9].

Se determinaron los pulsos de inundación a partir de las categorías de amenaza que usa la CAR (Corporación Autónoma Regional de Cundinamarca) referentes a la Tabla 1 donde clasifica las áreas dependiendo de la altura en que se encuentre el terreno determinando cuatro grupos de amenaza: muy alta, alta, media y baja. 
Siendo muy alta la zona con mayor riesgo de inundación.

Tabla 1. Clasificación de amenaza por evento de inundación según la CAR [8]

\begin{tabular}{|c|c|c|}
\hline AMENAZA & DESCRIPCIÓN & AREA \\
\hline BAJA & $\begin{array}{l}\text { Zonas con terrenos por } \\
\text { encima } 2548.3 \text { msnm, que } \\
\text { reporta la cota media } \\
\text { histórica reportada. Zonas } \\
\text { con índice bajo de } \\
\text { probabilidad de inundación, } \\
\text { pero no son exentas ser } \\
\text { ocupadas ante un invierno } \\
\text { extremo debido a sus } \\
\text { condiciones morfológicas }\end{array}$ & $\begin{array}{c}55.273 \\
\mathrm{Ha}\end{array}$ \\
\hline MEDIA & $\begin{array}{l}\text { Terrenos ubicados entre la } \\
\text { cota } 2540.5 \text { msnm y } 2548.3 \\
\text { msnm, reporta los niveles } \\
\text { máximos y } \\
\text { históricos. }\end{array}$ & $\begin{array}{c}57.781 \\
\mathrm{Ha}\end{array}$ \\
\hline ALTA & 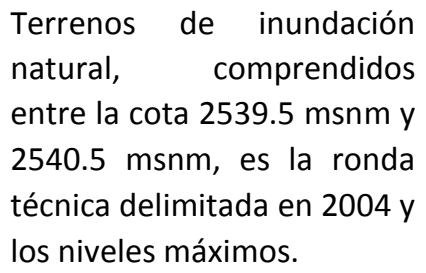 & $\begin{array}{c}49.505 \\
\mathrm{Ha}\end{array}$ \\
\hline MUY ALTA & $\begin{array}{l}\text { Las áreas de esta zona son } \\
\text { las aledañas al perímetro } \\
\text { de la laguna, que con } \\
\text { registros históricos ha } \\
\text { ocasionado procesos de } \\
\text { desecación de los bordes del } \\
\text { cuerpo hídrico. Comprende } \\
\text { el nivel medio y la ronda } \\
\text { técnica delimitada por } \\
\text { INCODER en 2004. } \\
\text { Aproximadamente cotas } \\
2538.8 \text { a } 2539.5 \mathrm{msnm} \text {. }\end{array}$ & $\begin{array}{c}0.9897 \\
\mathrm{Ha}\end{array}$ \\
\hline
\end{tabular}

ríos son afectadas por el cambio de niveles de agua que ocurren en la laguna [8].

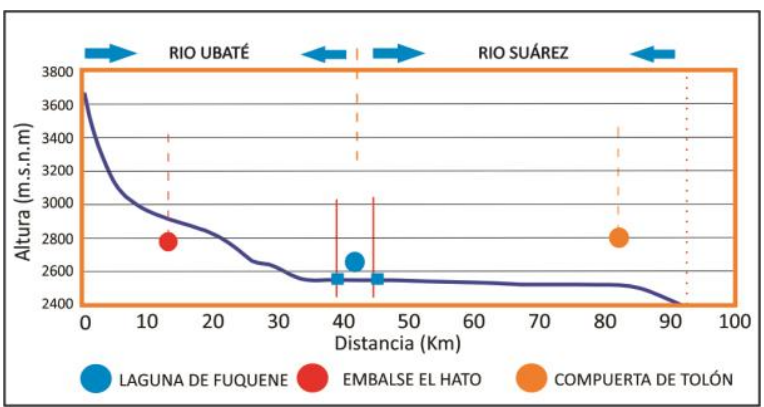

Figura 2. Perfil Río Ubaté, Laguna de Fúquene y Río Suárez [8].

El área de avenamiento total es $993 \mathrm{~km} 2$, como las lagunas no nacen pero si se alimentan, La laguna de Fúquene es alimentada principalmente por ríos y quebradas de la cuenca Ubaté - Fúquene: Honda, Tagua, Miña, Monroy y Sosiego. Estos entran a la laguna desde el oriente, por otro lado el río Fúquene descarga en la laguna desde el occidente. [8]

El rio Ubaté es el principal rio que la alimenta la Laguna y el flujo de su caudal en la llegada a la laguna ha sido regulado mediante la construcción de dos canales una a la izquierda y otro a la derecha llamado vallado madre, que tienen como objetivo principal en las épocas de invierno llevar aguas directamente a la Laguna sin ocupar el cauce principal del rio, pero la laguna solo tiene un desagüe natural, El rio Suarez, el cual es incapaz de transportar el excedente de líquido en épocas de lluvia por el poco desnivel en el desarrollo de más de 35 kilómetros. [1].

En la figura 3 se presenta el mapa de las principales ríos y quebrabas que alimentan la laguna, igualmente su única fuente de desagüe que es el rio Suarez.

En la Figura 2 se muestra el perfil longitudinal de la laguna que inicia con el Río Ubaté (principal afluente) hasta la confluencia con la laguna de Fúquene, en este mismo sector nace el río Suárez, el recorrido total es de $91,83 \mathrm{~km}$. Debido a esta configuración particular, las cuencas de estos dos 


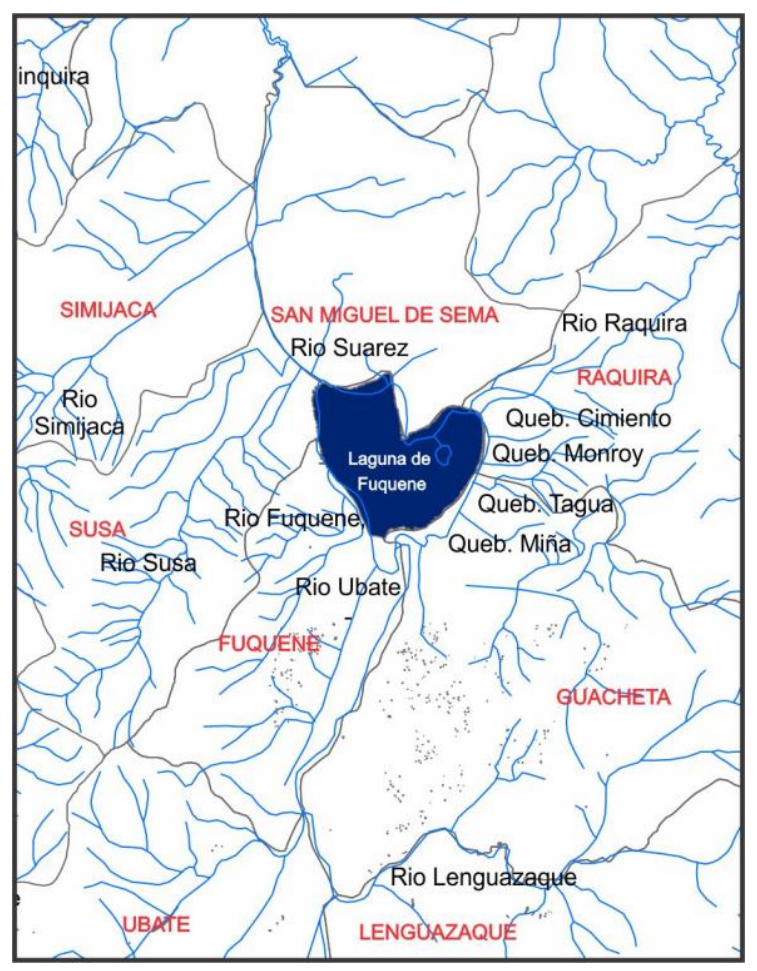

Figura 3. Ríos y quebradas principales que alimentan y descargan la Laguna de Fúquene.

\section{METODOLOGÍA}

En la metodología se explica las actividades realizadas para cumplimiento de los objetivos planteados inicialmente.

La primera fase fue la recolección de información de los niveles de agua de la Laguna de Fúquene, que son determinados por la estación limnimétrica Chalet Norte con código 2401720 la cual es monitoreada diariamente por Corporación Autónoma Regional de Cundinamarca (CAR), Los datos muéstrales se tomaron de la serie histórica de niveles de la Laguna desde 01 de enero de 1996 a 30 de abril del año 2017, con un total de 7791 datos muéstrales, teniendo un índice de confiabilidad del $95 \%$, Se da con el fin de obtener tendencias de inundación que puede llegar a tener la laguna, para lo cual se utilizó métodos estadísticos y probabilísticos que serán detallados a continuación.

Para el análisis de los datos se utilizó la base de datos Excel, Utilizando las herramientas de estadística (Media aritmética, máximos y mínimos) para analizar los datos de registros de los fenómenos hidrológicos pasados y hacer inferencias acerca de su comportamiento en el futuro, a través del método de la probabilidad de ocurrencia de inundación donde:

$P=m / n$

Donde:

$\mathrm{P}=$ probabilidad

$\mathrm{m}=$ número de orden

$\mathrm{n}=$ número total de casos

La probabilidad mide la mayor o menor posibilidad de que se dé un determinado resultado (suceso o evento) cuando se realiza un experimento aleatorio. Esta predicción es de carácter probabilístico y no determinístico.

Para completar los datos faltantes (72 días), Se usó un método de imputación sencillo, una vez teniendo completos los datos se da orden a los datos cronológicamente, que para cada fecha se registra el nivel de agua la Laguna.

Como segunda fase, se obtuvieron los resultados de pulsos de inundación, histogramas, curvas de permanencia, distribución temporal de los eventos de inundación de la laguna de Fúquene y frecuencia de los niveles del agua de la laguna de Fúquene; para los cuales se realizaron de la siguiente manera y se registran más adelante detalladamente en la sección de resultados.

Inicialmente se graficó la línea del comportamiento de los niveles de la laguna (fecha vs nivel de agua), denominada pulsos de inundación.

Para determinar el comportamiento que tuvo mensualmente la Laguna respecto a la cantidad de días que fue superado el nivel de referencia para cada una de las categorías de amenaza, se calcula la media mensual multianual, datos representados mediante en un histograma, Donde para cada categoría de amenaza está representada la cantidad de días promedio que superaron y no superaron el nivel de inundación referenciado para cada categoría de amenaza. 
Predecir la probabilidad de ocurrencia de inundación se utilizó una curva de permanencia que nos indica la probabilidad de que la Laguna supere cierto nivel de referencia, para el caso en estudio los cuatro niveles de amenaza( Muy alta, Alta, media y baja), para pulso presentado en cada nivel de referencia, se contabilizo los días continuos que han igualado o excedido el nivel de inundación, dando el valor de cero (0) aquellos días que no fue superado $o$ igualado el nivel de referencia evaluado. Posterior a esto se ordenaron los datos muéstrales de mayor a menor teniendo el dato mayor la menor probabilidad de ocurrencia, teniendo un numero de orden 1. Para el caso del número máximo de días continuos que ha permanecido inundado por superación de la cota de referencia a modo de ejemplo seria:

\section{$P=1 / 7791 \quad P=0,00012835 \quad P=0,012835 \%$}

Donde:

$\mathrm{P}=$ probabilidad

$\mathrm{m}=$ número de orden

$\mathrm{n}$ = número total de días evaluados

Lo que indica que existe una probabilidad de 0,012835\% que el valor número máximo de días que ha excedido o igualado el valor de referencia de cada categoría de amenaza ocurra.

Teniendo la probabilidad de ocurrencia de cada dato muestral se representan mediante una curva de permanecía (probabilidad de la permanencia vs Número de días permanecía de la inundación) para cada nivel de amenaza.

Para representar la probabilidad de ocurrencia de inundación y su nivel de agua promedio de permanencia para cada categoría de amenaza de forma mensual multianual se representa mediante el grafico de distribución temporal mensual multianual en donde las abscisas (eje $x$ ), representan los meses del año y (eje y) la para probabilidad de ocurrencia que se cómo define el cociente entre casos que la Laguna duro inundado en determinado mes y el total de días totales de cada mes evaluados.
Mediante el grafico de frecuencia de los niveles de agua se graficó la permanencia en días en determinado nivel de agua que pudo estar la Laguna, calculando su frecuencia absoluta y acumulada, mostrando los niveles en los cuales tuvo mayor y menor permanencia.

\section{RESULTADOS}

Se calculó el número de pulsos de inundación ocurridos en cada nivel de amenaza (Tabla 2), que han sucedido en la laguna de Fúquene desde 1 de enero de 1996 hasta el 30 de abril de 2017, con una muestra total de 7791 días analizados, de los cuales 72 días $(0,9 \%)$ de los datos totales no registraron medición de nivel de agua.

Los niveles de agua representados son respecto a la cota base de la Laguna 2537.72 msnm (cota 0 de la mira), que es la cota de referencia de la estación limnimetrica Chalet Norte, en el grafico se referencian los cuatro niveles de riesgo de inundación mostrados en la tabla 1 . Con las cotas de referencia y los niveles de agua registrados históricamente se pudo deducir cuantitativamente los pulsos de inundación presentados para cada uno de los 4 niveles de inundación, mostrados en la tabla 2, junto con datos relevantes de los pulsos en cada categoría de amenaza.

Mediante el pulso de inundación general (grafica de niveles de agua histórico) de la Laguna de Fúquene (Figura 4) puede inferir que los años con mayor variación en las categorías de amenaza son: 2006, 2010, 2011, 2012 y 2017, especialmente en el año 2011 con una cota de 2540,67 msnm en el mes de mayo, durante el mes de diciembre tuvo una cota de 2540,54 msnm.

En la Tabla 2 se relaciona los resultados obtenidos, se presenta media de los niveles máximos y mínimos, fecha del nivel máximo y mínimo registrado en el periodo de estudio y demás datos relevantes como se puede apreciar en la tabla. 
Tabla. 2 Resumen del número de pulsos de inundación según categoría de amenaza y datos relevantes

\begin{tabular}{|c|c|c|c|c|}
\hline AMENAZA & ZONA MUY ALTA & ZONA ALTA & ZONA MEDIA & ZONA BAJA \\
\hline \multicolumn{5}{|l|}{ Identificación de la zona } \\
\hline \multirow{3}{*}{ Nivel de Amenaza (msnm) } & 2538,8 & 2539,5 & 2540,5 & Superior a \\
\hline & a & a & a & 2548,3 \\
\hline & 2539,5 & 2540,5 & 2548,3 & \\
\hline & 1,08 & 1,78 & 2,78 & Superior a \\
\hline Nivel de amenaza/sobre cota cero $-2537,32$ & a & $\mathrm{a}$ & a & 10,58 \\
\hline msnm (m) & 1,78 & 2,78 & 10,58 & \\
\hline Área amenazada (Ha) & 0,99 & 49,50 & 57,78 & 55,2 \\
\hline Numero de pulsos & 34 & 15 & 3 & 0 \\
\hline Media de los niveles máximos (m) & \multicolumn{4}{|c|}{$1,711 \mathrm{~m}$ sobre cota cero } \\
\hline Media de los niveles mínimos (m) & \multicolumn{4}{|c|}{$0,774 \mathrm{~m}$ sobre cota cero } \\
\hline Fecha del nivel máximo & \multicolumn{4}{|c|}{$20 / 05 / 2011$} \\
\hline Fecha del nivel mínimo & \multicolumn{4}{|c|}{$07 / 12 / 2015$} \\
\hline Meses con Niveles de agua más Altos & \multicolumn{4}{|c|}{ Enero, Mayo, Junio, Julio, Noviembre, Diciembre } \\
\hline Meses con niveles de agua mas bajos & \multicolumn{4}{|c|}{ Febrero, Marzo, Abril, Agosto, Septiembre, Octubre } \\
\hline
\end{tabular}

En la Figura 4 se muestran los pulsos de inundación y su duración temporal durante el periodo de estudio, con referencia a la cota cero y las diferentes categorías de amenaza.

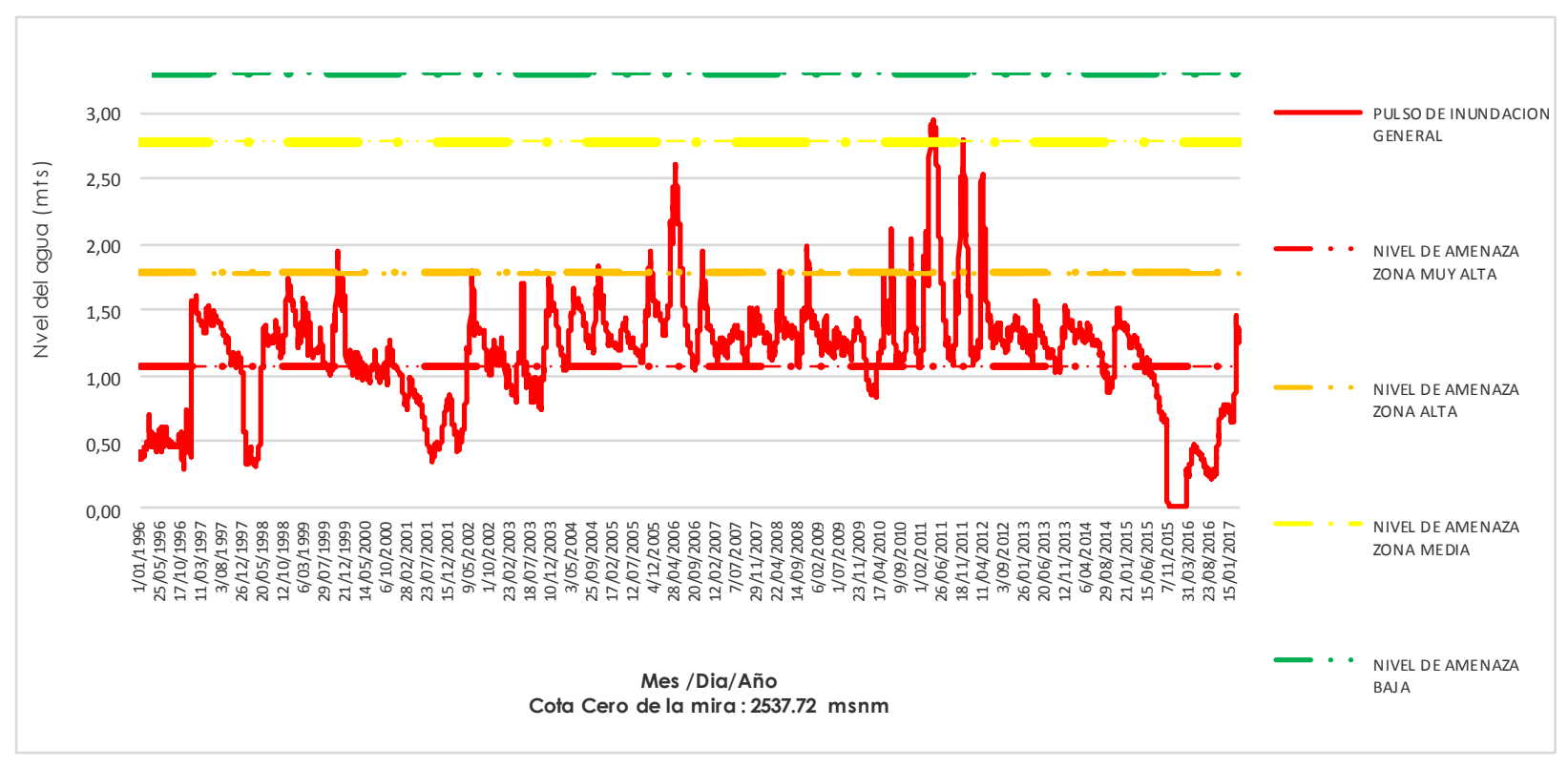

Figura 4. Pulsos de inundación: categoría de amenaza muy alta $(1,08 \mathrm{~m}$ por encima de la cota cero, $2.538,8$ $\mathrm{msnm})$, categoría de amenaza alta (1,78 m por encima de la cota cero, $2.539,5 \mathrm{msnm}$ ), categoría de amenaza media $(2,78 \mathrm{~m}$ por encima de la cota cero, $2.540,5 \mathrm{msnm})$ y categoría de amenaza baja $(10,58 \mathrm{~m})$ por encima de la cota cero, $2.548,3 \mathrm{msnm}$. 
En la Figura 5 se exhiben los histogramas mensuales multianuales de los niveles de agua que superan las categorías de amenaza
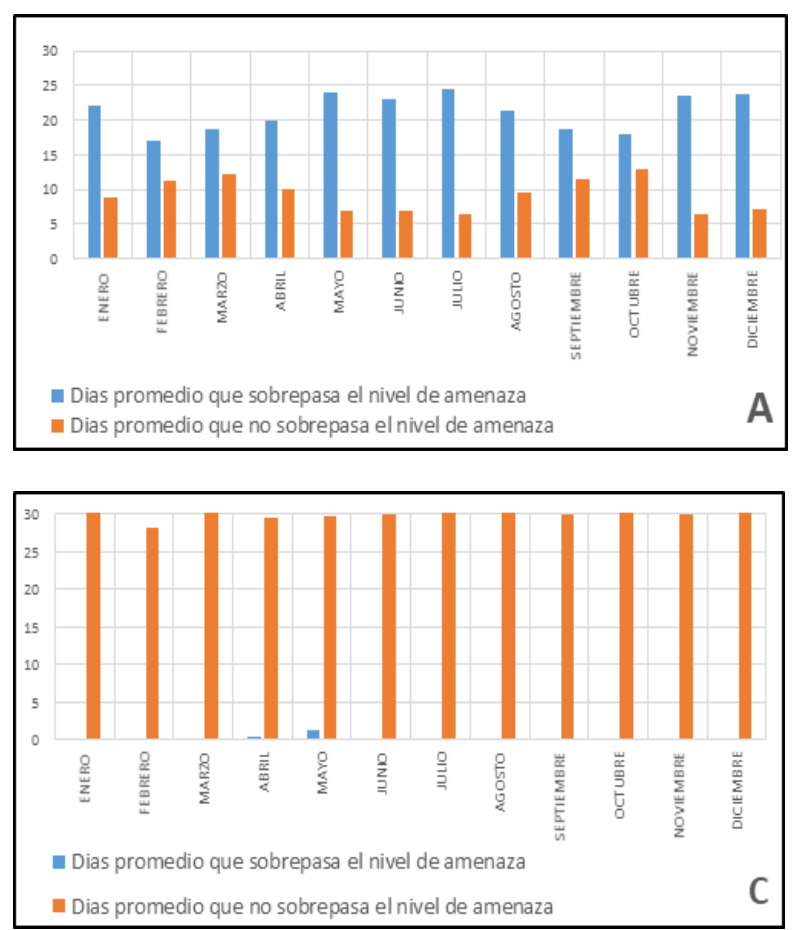

en días consecutivos según la cota de referencia.
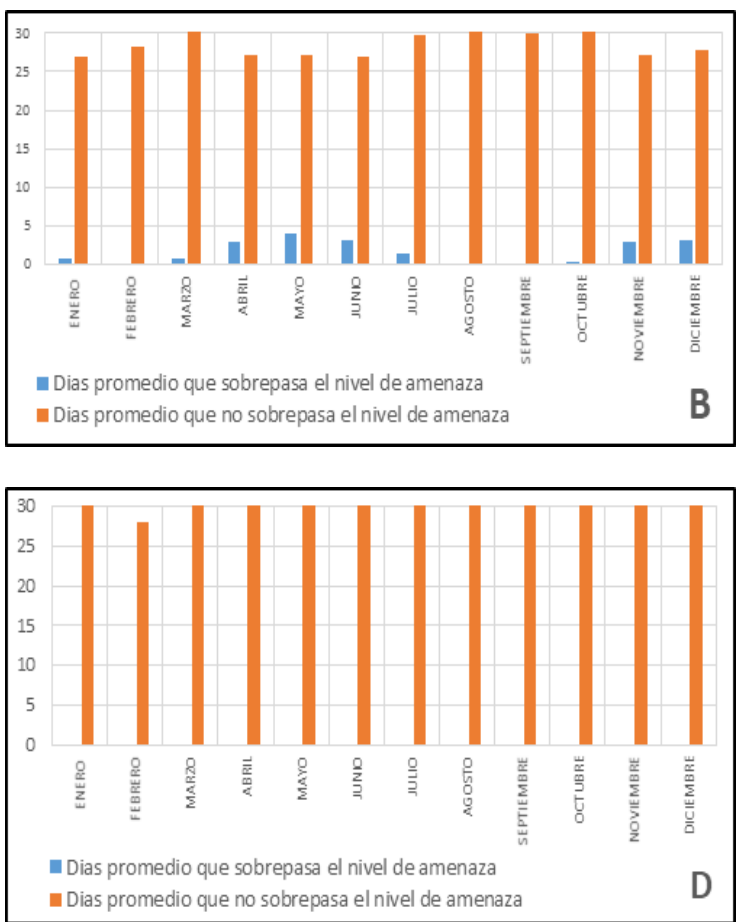

Figura 5. Histogramas (A) categoría de amenaza muy alta (1,08 m por encima de la cota cero, $2.538,8 \mathrm{msnm})$. (B) categoría de amenaza alta (1,78 m por encima de la cota cero, $2.539,5 \mathrm{msnm})$. (C) categoría de amenaza media $(2,78$ $\mathrm{m}$ por encima de la cota cero, $2.540,5 \mathrm{msnm})$. (D) categoría de amenaza baja (10,58 m por encima de la cota cero, $2.548,3 \mathrm{msnm})$

Los terrenos aledaños a la Laguna de Fúquene y que comprenden su altitud entre los niveles de referencia en la zona de amenaza muy alta, se estimaron 34 pulsos de inundación con un periodo máximo de permanencia de 885 y 472 días. Para el siguiente nivel determinado como zona alta se estimaron 15 pulsos inundación con un periodo máximo de permanencia de 89 y 86 días.

En el 2011 en la laguna ocurrió un mayor nivel de agua que supero la zona de categoría de amenaza media, en tres ocasiones, del 22 de abril al 10 de mayo, del 15 de abril al 1 de junio y por último el 15 de diciembre, con una duración de inundación de 19,18, y 1 días respectivamente.

4.1 Curvas de Permanencia en la Laguna de Fúquene.
La inundación es evento natural y frecuente que se genera en las corrientes de agua, como consecuencia de una alta precipitación, al rebasar el alcance de retención del suelo y de los cauces, invaden e inundan llanuras de inundación, en general, afectan aquellas áreas aledañas a los cuerpos de agua. [10].

En la Figura 6 se representa la curva de permanencia de la categoría de amenaza muy alta donde el mayor evento de inundación de esta categoría fue de 885 días continuos durante el periodo de estudio y ocurrió en el periodo de Abril de 2004 y Septiembre de 2006, dicho evento se caracterizó por un descenso del nivel de agua por 6 días y volvió a permanecer inundado entre 300 y 400 días consecutivos excediendo el nivel de referencia de la categoría de amenaza muy alta, la 
que tiene una zona de afectación de aproximadamente $1(\mathrm{Ha})$.

En la Figura 7, se representa la curva de permanencia de la categoría de amenaza alta donde el mayor evento de inundación de esta zona fue de 89 días continuos durante el periodo de estudio y ocurrió en el año 2011 donde la laguna por efectos de la temporada invernal mantuvo las mayores excedencias en la cota media, afectando las zonas aledañas.
Para las categorías de amenaza media y baja no se construyeron curvas de permanencia, ya que, se identificaron menos de 5 pulsos, razón por lo cual, en el método de la curva de permanencia no se puede graficar los pulsos con menos de $1 \%$ de probabilidad, lo anterior no significa que las zonas no sean vulnerables, ya que, se identificó que la categoría de amenaza media uno de los pulsos presento 19 días continuos de permanencia, en el año 2011.

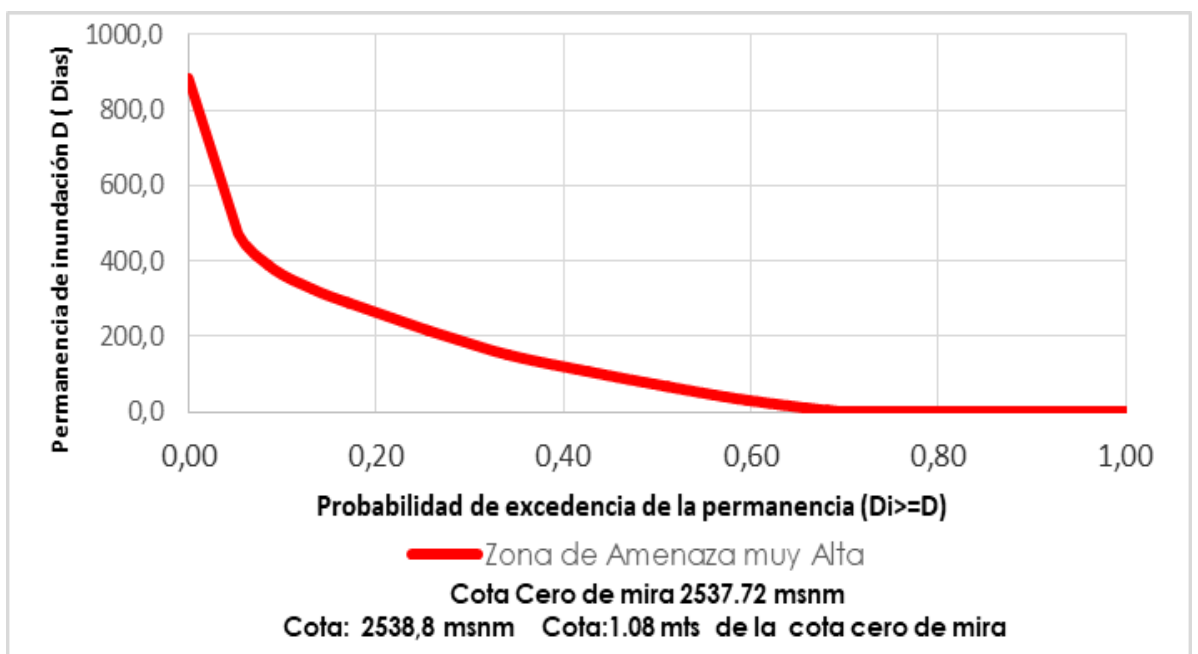

Figura 6. Curva de Permanencia de la Laguna de Fúquene (ZMA).

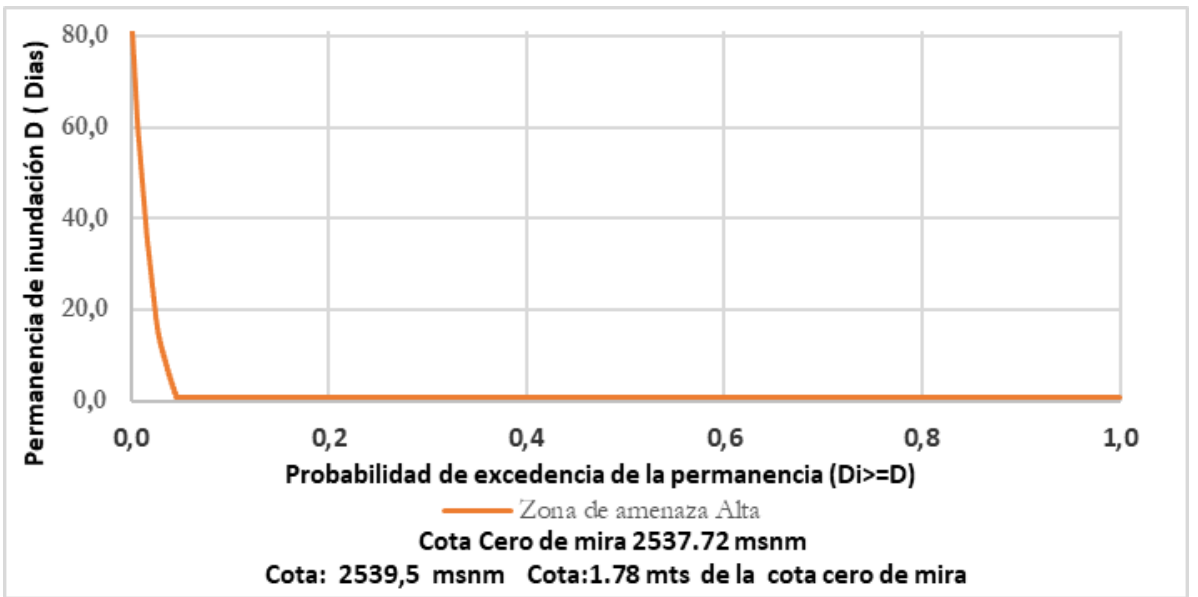

Figura 7. Curva de Permanencia de la Laguna de Fúquene (ZA).

\subsection{Distribución Temporal de las Inundaciones en la Laguna de Fúquene}

La frecuencia de las inundaciones en el tiempo, tiene un valor informativo fundamental en diferentes ámbitos como lo pueden ser la construcción, agricultura y ganadería entre otros; debido a que son factores donde es necesario tener planificada la producción y prevención por los efectos climatológicos. [10]. 
Para el periodo de tiempo en estudio, se reporta que los meses con mayor probabilidad de inundación son: Mayo a Julio, así mismo con una probabilidad menor en los meses de Febrero, Septiembre y Octubre.

En la Figura 8 se representan los meses que tienen mayor probabilidad de inundación en el año (diagrama de barras), junto con los niveles medios mensuales multianuales de agua (línea continua).
En la Figura 9 y la Tabla 3 se muestra y relaciona la frecuencia absoluta y acumulada de los niveles de agua diarios consecutivos, con esta grafica se analizan la variabilidad de las cotas de la niveles de agua de la Laguna de Fúquene con una mayor frecuencia concentrada entre $2538,8 \mathrm{msnm}$ y 2539,1 msnm ratificando los resultados obtenidos de los pulsos de inundación y las curvas de permanencia para cada zona de amenaza.

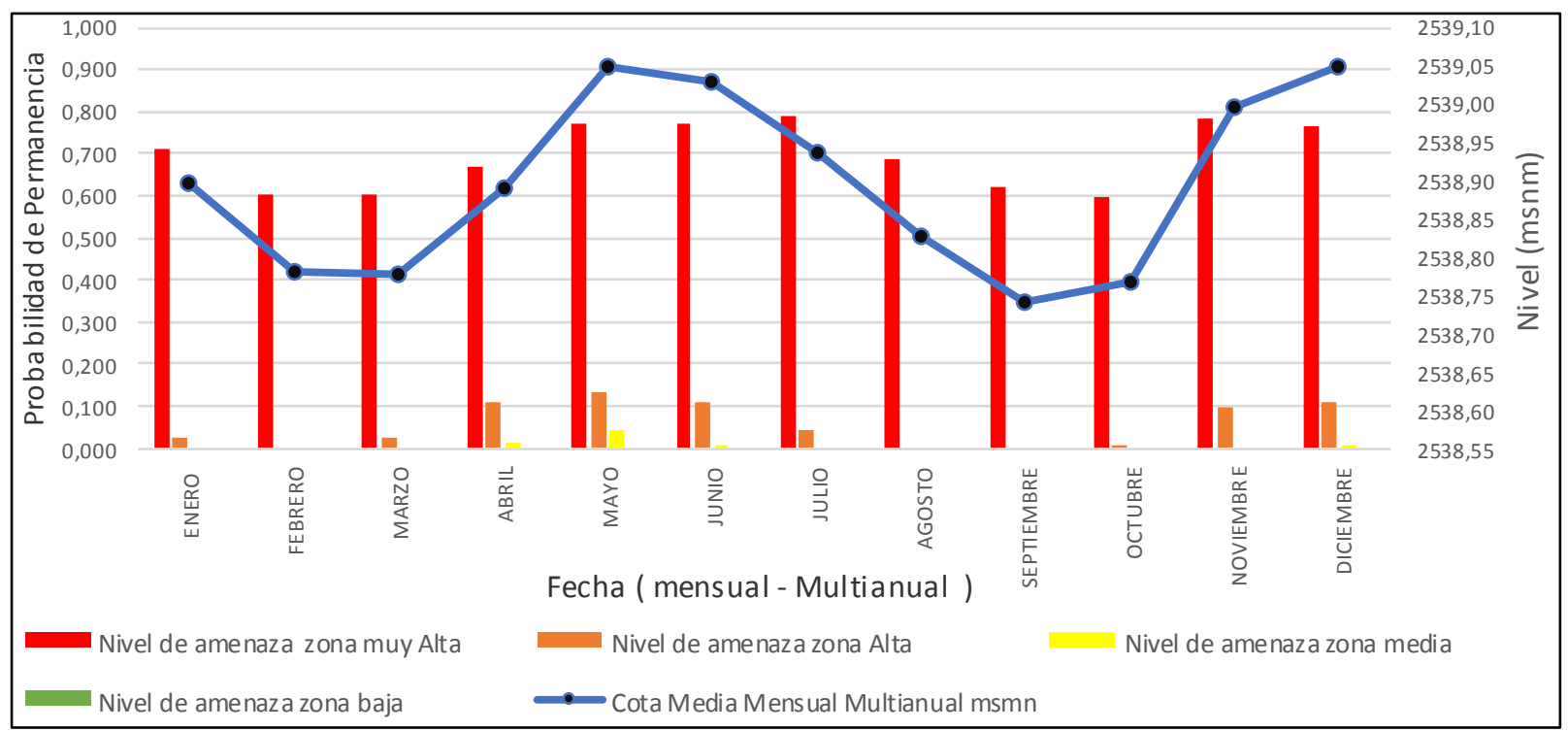

Figura 8. Distribución temporal de los eventos de inundación de la Laguna de Fúquene.

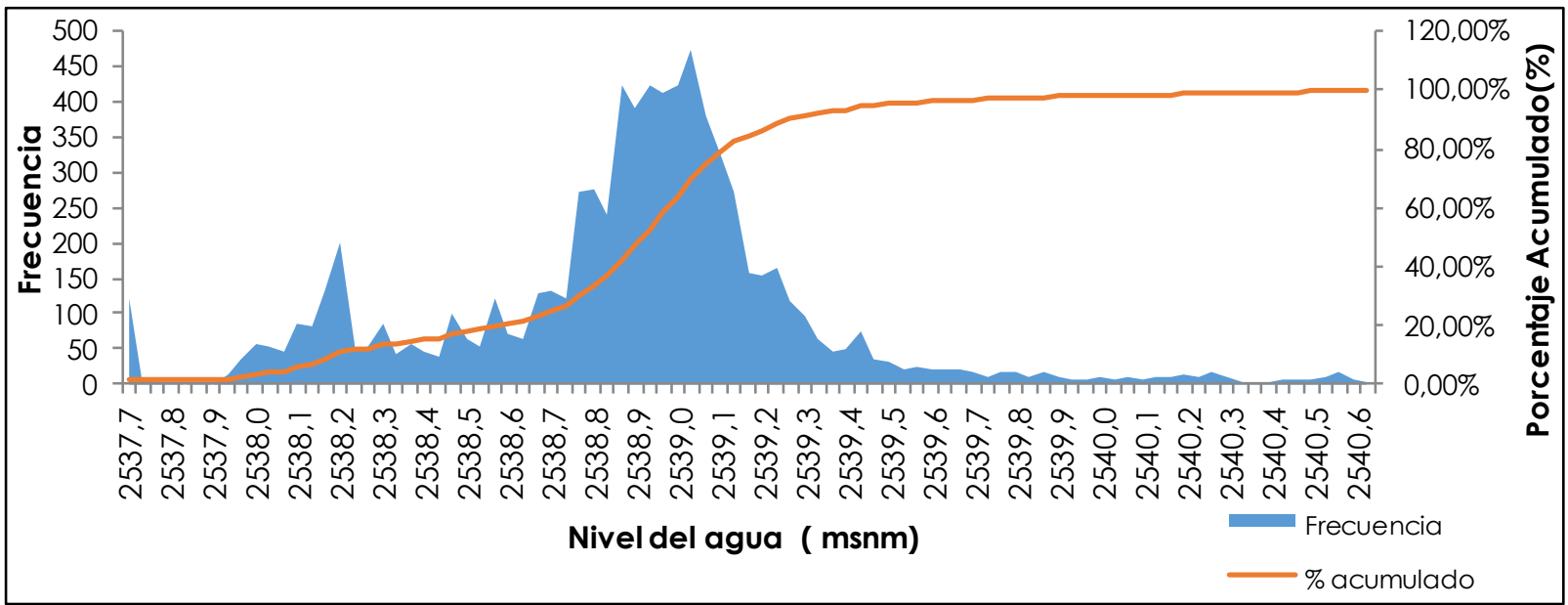

Figura 9. Frecuencia de los niveles del agua de la Laguna de Fúquene. 
Tabla 3. Descripción de la Frecuencia de los niveles del agua de la Laguna de Fúquene.

\begin{tabular}{|c|c|c|c|c|c|}
\hline \multirow{2}{*}{ MES } & \multicolumn{4}{|c|}{ AMENAZA } & \multirow{2}{*}{$\begin{array}{c}\text { Cota } \\
\text { Media } \\
\text { Mensual } \\
\text { multianua } \\
\text { (msnm) }\end{array}$} \\
\hline & \begin{tabular}{c}
\multicolumn{2}{c}{ Muy } \\
alta (\%)
\end{tabular} & $\begin{array}{l}\text { Alta } \\
(\%)\end{array}$ & $\begin{array}{c}\text { Media } \\
(\%)\end{array}$ & $\begin{array}{c}\text { Baja } \\
(\%)\end{array}$ & \\
\hline Ene & 71,11 & 2,26 & 0,00 & 0,0 & 2538,9 \\
\hline Feb & 60,55 & 0,00 & 0,00 & 0,0 & 2538,7 \\
\hline Mar & 60,41 & 2,74 & 0,00 & 0,0 & 2538,7 \\
\hline $\mathrm{Abr}$ & 66,82 & 10,67 & 1,50 & 0,0 & 2538,8 \\
\hline May & 77,42 & 13,23 & 4,35 & 0,0 & 2539,0 \\
\hline Jun & 77,14 & 10,83 & 0,17 & 0,0 & 2539,0 \\
\hline Jul & 78,96 & 4,35 & 0,00 & 0,0 & 2538,9 \\
\hline Agost & 68,97 & 0,00 & 0,00 & 0,0 & 2538,83 \\
\hline Sept & 62,06 & 0,00 & 0,00 & 0,0 & 2538,7 \\
\hline Oct & 59,90 & 0,48 & 0,00 & 0,0 & 2538,7 \\
\hline Nov & 78,57 & 10,0 & 0,00 & 0,0 & 2539,0 \\
\hline Dic & 76,80 & 10,65 & 0,16 & 0,0 & 2539,05 \\
\hline
\end{tabular}

De la tabla 3 se puede inferir que el régimen temporal de los niveles de permanencia (pulsos de inundación) tiene mayor influencia en los meses de Abril a Junio.

\section{CONCLUSIONES}

Una vez realizado el estudio de los pulsos de inundación se detectó una reiterada inundación para los niveles de amenaza muy alta y alta, en los meses de Mayo, Junio, Julio, Noviembre y Diciembre.
El nivel de amenaza muy alta se inundó todos los años de estudio menos los años 1996, 2001 y 2016, lo que demuestra que dicha zona tiene una alta probabilidad de inundarse por lo menos una vez al año. Un comportamiento similar puede ajustarse a la categoría de amenaza alta, el área afectada por los eventos de inundación es de 50,59 Ha.

A partir de los registros diarios de los niveles de agua de la estación limnimétrica Chalet Norte operada por la CAR, se determinó que las zonas aledañas a la Laguna entre las cotas 2538.8 a $2539.5 \mathrm{msnm}$ poseen un alto riesgo de inundación, con una probabilidad del $40 \%$ que la Laguna supere la cota de referencia de inundación con una duración de 120 días consecutivos.

Ante la inundación que produce la Laguna de Fúquene en las zonas aledañas entre las cotas mencionadas, se recomienda a las autoridades pertinentes iniciar una recuperación de las zonas aledañas o en su defecto recuperar gran parte del espejo de agua para así recobrar la capacidad de carga hídrica de la laguna, dada la gran importancia que representa para los Departamentos de Boyacá, Cundinamarca y Santander.

El nivel máximo de la Laguna se presentó el 20 y 21 de mayo de 2011, superando el nivel medio de amenaza, durante el mismo año el nivel de la Laguna sobrepaso el nivel medio de amenaza en tres ocasiones, la primera del 22 de abril al 10 de mayo, la segunda del 15 de mayo al 1 de junio y la tercera el 18 de diciembre, con una duración de 19, 18 y 1 días respectivamente por encima del nivel medio de amenaza. Siendo el año 2011 con mayor afectación de área inundada, que coincide con el fenómeno de la Niña de 2010-2011 que se vivió en algunas regiones del país [11]. Lo que demuestra que ante un futuro fenómeno de la Niña en la región muy posiblemente las áreas o terrenos cercanos a la Laguna ubicados entre la cota $2540.5 \mathrm{msnm}$ (o menor a esta cota) y $2548.3 \mathrm{msnm}$ (cotas de nivel medio de amenaza), se verán afectados por inundación.

Los datos suministrados pueden ser un aporte para estudiar la relación que pueden tener diferentes tipos de afectaciones en la zona como: producción 
de algún tipo de siembra, afectación de las fincas aledañas a la Laguna, desaparición de alguna especie animal que tenía su hábitat en la zona, plantas que por los cambios de niveles han desaparecido con el trascurrir de los años y proliferación de vectores.

Además se deben plantear estudios de gestión de riesgo y de ordenamiento territorial con el fin de disminuir las afectaciones por eventos de inundaciones en las zonas con categorías de amenaza muy alta y alta.

\section{REFERENCIAS}

[1] O. W. Cabrero, "La Laguna de Fúquene". Boletín de la Sociedad Geográfica de Colombia, vol. 15, no. 53, pp. 1-20, 1957.

[2] Fundación Humedales, "Complejo De Lagunas De Fúquene, Cucunubá Y Palacio" 2017 Disponible en http://www.ecoindex.org/search/pdfs/796report_1. pdf.

[3] C. J . P. Espitia, "Análisis de la Aplicación del Enfoque Ecosistémico en la Estrategia de Manejo Ambiental de la Cuenca Ubaté-Suárez Implementada en la Laguna de Fúquene durante el período 20072009", Tesis pregrado, Facultad de Relaciones Internacionales, Estrategia y Seguridad., Universidad Militar Nueva Granada., Bogotá, Colombia, 2010.

[4] Conpes, "Documento conpes 3451 Estrategia para el Manejo Ambiental de la Cuenca UbatéSuárez", Consejo Nacional de Política Económica y Social República de Colombia Departamento Nacional de Planeación, Bogotá DC., 2006.

[5] J. J. Neiff, "El régimen de pulsos en ríos y grandes humedales de Sudamérica". Tópicos sobre humedales subtropicales y templados de Sudamérica, vol. 229, pp. 99-103, 1999.

[6] V.M. Ponce, "El balance de nutrientes bajo el pulso de inundación" [online]. California: Universidad
Estatal de San Diego, 2017 Disponible en http://ponce.sdsu.edu/el_balance_de_nutrientes_ba jo_el_pulso_de_inundacion.html

[7] W. Junk, P.B. Bayley, and R.E. Sparks. "The flood pulse concept in river-floodplain systems", Proceedings of the International Large River Symposium (LARS). Dodge, D.P. ed. Canadian Special Publication of Fisheries and Aquatic Sciences, vol. 106, pp. 110-127, 1989.

[8] CAR. "Plan de Ordenamiento de la Cuenca de los Ríos Ubaté y Suarez. Diagnóstico, prospectiva y formulación de la cuenca hidrográfica de los Ríos Ubaté y Suárez, Corporación Autónoma Regional de Cundinamarca CAR, Bogotá, 2006.

[9] L. Franco-Vidal, C. A. Ruiz-Agudelo, J. Delgado, G. Andrade y A. Guzmán, "Interacciones socioecológicas que perpetúan la degradación de la laguna de Fúquene, Andes orientales de Colombia." Ambiente y Desarrollo, vol. 19, 37, pp. 49-66, Julio-Diciembre, 2015.

DOI:https://doi.org/10.11144/Javeriana.ayd1937.ispd

[10] C. Castaño-Uribe, R. Carrillo, \& F. Salazar, "Sistema de Información Ambiental de Colombia Tomo III. Perfil del estado de los recursos naturales y del medio ambiente en Colombia 2001", IDEAM. Min. Medio Ambiente, Bogotá, Julio 2002.

[11] N.A. Abril-Gonzalez, E.G. Amaya-Reyes, H.A. Fonseca-Peralta, Evaluación de amenazas por movimientos en masa causados en Jericó Boyacá, durante el periodo invernal de 2011,"Revista Ingeniería, Investigación y Desarrollo", 14(1), pp.613, Enero 2014.

DOI: https://doi.org/10.19053/1900771X.5115 